\title{
Lipoid secretion by epidermis of bare skin from the head of the Indian White Ibis, Threskiornis Melanocephala
}

\author{
G.K. MENON, R.V. SHAH and M.B. JANI
}

That avian epidermis produce lipoid substance (s) functionally akin to the sebum of mammals, is well recognised by now (Lucas, 1968; Matoltsy, 1969; Lucas and Stettenheim, 1972; Shah and Menon, 1972). Such production of lipoid substance $(s)$ is restricted to the epidermis of the interplumar regions, while in the feather follicles, the product of epithelial cell activity is usually a structural protein-keratin (exceptions are the fat quills described by Lucas and Stettenheim, 1972). In many species of birds, with attainment of maturity, initially feathered regions of the body like the head and neck lose feathers and become bare. The only available information on histophysiological changes occuring in the avian integument accompanying such transformations comes from Shah et al: (1977), who reported an enhanced lipoid secretion in the secondarily formed capital apterium of the Painted Stork, Ibis leucocephalus. The present communication reports findings on epidermal lipoid secretion in the Indian White Ibis (Threskiornis melanocephala), another avian species wherein adults have secondarily formed bare areas of skin on their head and neck regions.

\section{Material and methods}

Adult birds that died accidentally or by natural causes in the Sayaji Zoological Garden adjacent to the University campus, Baroda, and immediately made available to us from time to time by courtsey of the Superindentant, were used for the present observations. Pieces of skin were excised immediately from the capital as well as from the cervical regions. Frozen sections of 10 to $15 \mu$ thickness were cut on a cryostat microtome They were fixed in Baker's calcium formol and stained with Fat Red 7 B (Sigma Chemical Company, U.S.A.), aqueous Nile Blue Sulphate or Sudan Black B (as per methods described by Pearse, 1960), for histochemical demonstration of various types of lipids in the skin. Stained sections were mounted in glycerine jelly and observed under the microscope. Pieces of skin fixed in Buoin's fixative were used for preparation of paraffin sections for routine histological studies. Unfixed sections treated with a $1: 2(\mathrm{~V} / \mathrm{V})$ mixture of

Division of Avian Biology, Department of Zoology, Faculty of Science, M.S. University of Baroda, Baroda India. 
chloroform: methanol, prior to staining were used as controls to check the authenticity of histochemical methods.

\section{Observations and discussion}

The stratified epidermis of the secondarily bare skin has a fairly heavy concentration of melanin pigment. Melanocytes are discernible in the dermis subjacent to the epidermis. No trace of feather follicles are seen in these areas of the skin. Lipid content of the dermis is poorer than that of the epidermis which has a great deal of neutral lipids in its cells. On applying slight pressure to the mounted sections, oil droplets can be squeezed out of the epidermal layer. These are neither artifacts nor derived from the dermal fat depots, as they are always stained red /pink with Fat Red 7 B and aqueous Nile Blue Sulphate (specific staining properties of triglycerides) and as no adipose tissue cells are seen immediately subjacent to the epidermis. This indicates the high degree of lipoid synthesis and its accumulation in the epidermis in the secondarily bare skin of capital and cervical regions of the Indian White Ibis. By desquamation of the outermost lipid loaded epidermal cells, the lipoid substance ( $s$ ) gets spread over the skin, the whole of the bare skin functioning much like a holocrine gland. This observation also confirms our earlier contention that apteric regions that are secondarily formed as a result of permanant loss of feathers tend to show an enhanced secretory activity. Our contention is further borne out by the observations of Lucas (personal communication) that in pheasants too, the non-feather epidermis has a much higher lipid content than in the feather tracts.

Feather loss and formation of an apterium on the head and neck regions in the White Ibis seem to be functionally correlated with the habitat and feeding habits of the birds. Ali (1956) reports that when these birds probe into the bottom mud in shallow water, the head is sometimes completely immersed momentarily. The oil bearing epidermis would then be of obvious adaptive value, preventing the skin from remaining water soaked, apart from maintaining its flexibility and integrity in spite of being exposed or uninsulated. This observation also underscores the fact that such functional modifications of the avian integumentary system are of more common occurrence than was hitherto suspected. The role of pigmentation of the bare skin in social communication and its significance in the social life of these birds also cannot be overlooked, considering that the same areas of skin are not coloured black when they were feathered in the subadults.

\section{References}

Ali, S. 1956. The birds of Gujrat. J. Bombay. Nat. Hist. Soc. 52: 374-458.

Lucas, A.M. 1968. Lipoid secretion in the avian epidermis (Abstract). Anat. Rec. 60: 386-387. 
Lucas, A.M. 1978. Personal communication.

Lucas, A.M. and Stettenheim, P.R. 1972. Avian Anatomy: Integument. Agriculture Handbook 362, U.S. Dept. Agriculture, Washinton, D.C.

Matoltsy, G.A. 1969. Keratinization of the avian epidermis: An ultrastructural study of the new born chick skin. J. Ultrastructure Research. 29 : 438-458.

Pearse, A.G.E. 1960. Histochemtstry, theoretical and applied. 2nd Ed. Little, Brown \& Co, Boston.

Shah, R.V. and Menon, G.K. 1972. Histochemical studies on pigeon definitive feathers during post-hatching, induced and regenerative modes of development. III. Lipids, lipase and $\beta$-hydroxybutyrate dehydrogenase. Pavo $10: 30-42$.

Shah, R.V., Menon, G.K., Desai, J.H. and Jani, M.B. 1977. Feather loss from capital tracts of Painted Storks related to growth and maturity. I. Histophysiological changes and lipoid secretion in the integument. J. Anim. Morphol. Physiol. 24: 99-107. 

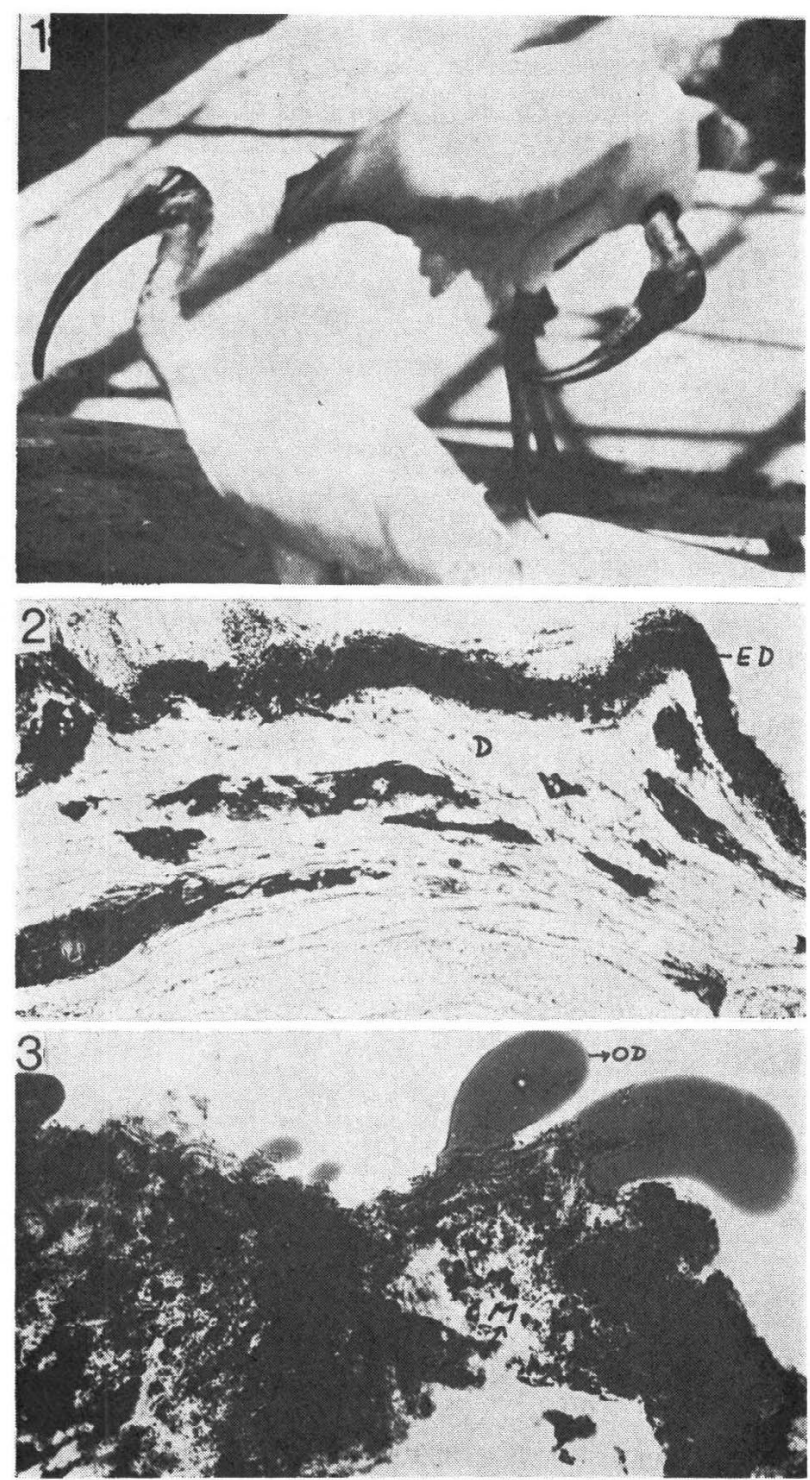

Fig. 1. Subadult (left) and adult (right) White Ibis. Notice feathered head of the subadult and black skin on head and neck of the adult.

Fig. 2. Vertical section of bare skin from the head of adult White Ibis stained for demonstrating neutral lipids (Stain-Fat Red 7 B)

Fig. 3. Enlarged view of epidermis showing lipid droplets oozing out after application of slight pressure to the slide. Dark black granules (M) are of melanin pigment.

Abbreviations

D-Dermis, ED-Epidermis, M-Melanin granules, OD-Oil droplet 